\title{
Combined radiotherapy and concurrent tumor treating fields (TTFields) for glioblastoma: Dosimetric consequences on non-coplanar IMRT as initial results from a phase I trial
}

N. Guberina', C. Pöttgen', S. Kebir², L. Lazaridis², C. Scharmberg', W. Lübcke', M. Niessen', M. Guberina', B. Scheffler ${ }^{3,4}$, V. Jendrossek ${ }^{5}$, R. Jabbarli ${ }^{6}$, D. Pierscianek ${ }^{6}$, U. Sure ${ }^{6}$, T. Schmidt ${ }^{2}$, C. Oster ${ }^{2}$, P. Hau ${ }^{7}$, A. L. Grosu ${ }^{8,9}$, M. Stuschke ${ }^{1,4^{*}}$, M. Glas ${ }^{2,4^{*}}$, Y. Nour ${ }^{1+}$ and L. Lüdemann ${ }^{1 \dagger}$

\begin{abstract}
Background: Glioblastoma is a rapidly proliferating tumor. Patients bear an inferior prognosis with a median survival time of 14-16 months. Proliferation and repopulation are a major resistance promoting factor for conventionally fractionated radiotherapy. Tumor-Treating-Fields (TTFields) are an antimitotic modality applying low-intensity (1-3 V/ $\mathrm{cm})$, intermediate-frequency $(100-300 \mathrm{kHz})$ alternating electric-fields. More recently interference of TTFields with DNAdamage-repair and synergistic effects with radiotherapy were reported in the preclinical setting. This study aims at examining the dosimetric consequences of TTFields applied during the course of radiochemotherapy.

Methods: Cone-beam-computed-tomography (CBCT)-data from the first seven patients of the PriCoTTF-phase-I-trial were used in a predefined way for dosimetric verification and dose-accumulation of the non-coplanar-intensitymodulated-radiotherapy (IMRT)-treatment-plans as well as geometric analysis of the transducer-arrays by which TTFields are applied throughout the course of treatment. Transducer-array-position and contours were obtained from the low-dose CBCT's routinely made for image-guidance. Material-composition of the electrodes was determined and a respective Hounsfield-unit was assigned to the electrodes. After 6D-fusion with the planning-CT, the dose-distribution was recalculated using a Boltzmann-equation-solver (Acuros XB) and a Monte-Carlo-dose-calculation-engine.

(Continued on next page)
\end{abstract}

\footnotetext{
* Correspondence: Martin.Stuschke@uk-essen.de; Martin.Glas@uk-essen.de

${ }^{\dagger}$ Y. Nour and L. Lüdemann contributed equally to this work.

${ }^{1}$ Department of Radiotherapy, West German Cancer Center, University

Hospital Essen, University of Duisburg-Essen, Hufelandstraße 55, 45147 Essen,

Germany

${ }^{2}$ Division of Clinical Neurooncology, Department of Neurology and West

German Cancer Center, University Hospital Essen, University of

Duisburg-Essen, Hufelandstraße 55, 45147 Essen, Germany

Full list of author information is available at the end of the article
}

C C The Author(s). 2020 Open Access This article is licensed under a Creative Commons Attribution 4.0 International License, which permits use, sharing, adaptation, distribution and reproduction in any medium or format, as long as you give appropriate credit to the original author(s) and the source, provide a link to the Creative Commons licence, and indicate if changes were made. The images or other third party material in this article are included in the article's Creative Commons licence, unless indicated otherwise in a credit line to the material. If material is not included in the article's Creative Commons licence and your intended use is not permitted by statutory regulation or exceeds the permitted use, you will need to obtain permission directly from the copyright holder. To view a copy of this licence, visit http://creativecommons.org/licenses/by/4.0/ The Creative Commons Public Domain Dedication waiver (http://creativecommons.org/publicdomain/zero/1.0/) applies to the data made available in this article, unless otherwise stated in a credit line to the data. 
(Continued from previous page)

Results: Overdosage in the scalp in comparison to the treatment plan without electrodes stayed below $8.5 \%$ of the prescribed dose in the first $2 \mathrm{~mm}$ below and also in deeper layers outside $1 \mathrm{~cm}^{2}$ at highest dose as obtained from dose-volume-histogram comparisons. In the clinical target volume (CTV), underdosage was limited to $2.0 \%$ due to dose attenuation by the electrodes in terms of D95 and the effective-uniform-dose. Principal-component-analysis (PCA) showed that the first principal-position-component of the variation of repeated array-placement in the direction of the largest variations and the perpendicular second-component spanning a tangential plane on the skull had a standard deviation of $1.06 \mathrm{~cm}, 1.23 \mathrm{~cm}, 0.96 \mathrm{~cm}$, and $1.11 \mathrm{~cm}$ for the frontal, occipital, left and right arrays for the first and 0.70 $\mathrm{cm}, 0.71 \mathrm{~cm}, 0.79 \mathrm{~cm}$, and $0.68 \mathrm{~cm}$, respectively for the second-principal-component. The variations did not differ from patient-to-patient ( $p>0.8$, Kruskal-Wallis-tests). This motion led to a diminution of the dosimetric effects of the electrodes.

Conclusion: From a dosimetric point of view, dose deviations in the CTV due to transducer-arrays were not clinically significant in the first 7 patients and confirmed feasibility of combined adjuvant radiochemotherapy and concurrent TTFields.

PriCoTTF Trial: A phase I/II trial of TTFields prior and concomitant to radiotherapy in newly diagnosed glioblastoma. DRKS-ID: DRKS00016667.

Date of Registration in DRKS: 2019/02/26.

Investigator Sponsored/Initiated Trial (IST/IIT): yes.

Ethics Approval/Approval of the Ethics Committee: Approved.

(leading) Ethics Committee Nr.: 18-8316-MF, Ethik-Kommission der Medizinischen.

Fakultät der Universität Duisburg-Essen.

EUDAMED-No. (for studies acc. to Medical Devices act): CIV-18-08-025247.

Keywords: Dosimetry, Non-coplanar IMRT, Glioblastoma, TTFields

\section{Background}

Glioblastoma (World Health Organization grade IV glioma [1]) is a rapidly proliferating tumor [2, 3]. Despite a trimodal approach, overall survival and progression free survival rates remain low [4-8]. Proliferation and repopulation are a major resistance promoting factor for conventionally fractionated radiotherapy [9]. Therefore, multimodal treatment is at present regarded as the best approach for handling this most common, aggressive brain tumor entity of adulthood.

Alongside with other established treatment options for glioblastoma (GBM) patients such as radiotherapy, surgical resection and temozolomide (TMZ) chemotherapy [10], Tumor Treating fields (TTFields) are a recently established modality reported as an effective maintenance therapy prolonging progression-free, overall and long-term survival when applied after radiochemotherapy together with TMZ in a positive phase III trial [11]. Though formally regarded as a negative trial, TTFields showed some effectiveness in recurrent glioblastomas [12]. In GBM patients TTFields therapy at $200 \mathrm{kHz}$ (Optune ${ }^{\circledR}$ ) (manufacturer: Novocure $\mathrm{GmbH}$, Munich, Germany) is delivered by four transducer arrays that are applied to the patients scalp. Each transducer array is composed of 9 ceramic discs having very high capacity covered by hydrogel. The discs are connected by a flexible printed circuit board for better adaptation to the head curvature. Each array contains 8 temperature sensors that monitor skin temperature. Optune ${ }^{\bullet}$ was granted marketing authorization by the FDA in 2015 and is CE marked approved [13]. TTFields act antimitotic by applying low intensity $(1-3 \mathrm{~V} / \mathrm{cm})$, intermediate frequency (100-300 kHz) alternating electric fields to treat solid tumors $[14,15]$. More recently, interference of TTFields with DNA damage repair and synergistic effects with radiotherapy were reported in the preclinical setting [16-18]. Moreover, first experiences were reported on clinical application of TTFields in combination with radiotherapy. The phase I trial conducted by Grossman et al. in which transducer arrays were removed during application of radiotherapy, provided first indication that the combined therapy is feasible and safe [19].

Mechanisms of interaction with radiotherapy are inhibition of proliferation by a maximal absorption of induced power during the mitotic furrow $[14,20]$ as well as suppression of double strand break repair by thwarting homologous recombination and by down-regulation of genes within the BRCA1 pathway genes $[16,21]$. In addition, TTFields delay DNA damage repair following radiation treatment of glioma cells [16]. This led to the hypothesis that the maximum interaction between TTFields and radiation may be achieved by simultaneous application [15, 21]. Until now, there exist no data from clinical trials, which examined the simultaneous application of TTFields and concurrent radiochemotherapy after surgery. Therefore, the multicenter PriCoTTF phase I trial (European 
database on medical devices (Eudamed) CIV 18-08025247) has been initiated to analyze the feasibility and safety of radiochemotherapy concomitant to TTField treatment in a first step.

Here, we report on the dosimetric consequences of transducer arrays by which TTFields are applied during the course of radiotherapy in the first seven patients of that trial. Endpoints of analysis were the accumulated dose during the course of radiotherapy in the clinical target volume (CTV) and in the whole brain outside the target volume as well as in the skin, subcutaneous tissue and calvarial bone below the transducer arrays. In addition, the variation of position of the regularly replaced transducer arrays on the skin was analyzed over the radiotherapy series.

\section{Methods}

In a prospective study design the first seven patients of the PriCoTTF phase I trial (European database on medical devices (Eudamed) CIV 18-08-025247) treated at the University Hospital Essen between 10.07.2019 and 15.12.2019 were included in the present dosimetric analysis.

\section{Patients}

Patients with a newly diagnosed, histologically confirmed glioblastoma were eligible for study arm A with an age $\leq 70$ years and a Karnofsky performance status (KPS) $\geq 60 \%$ and for study arm B at an age $>70$ years and a KPS $\geq 50 \%$. Written informed consent was obtained from all patients prior to therapy. The local Ethics Committee approved the trial.

\section{Trial design}

This is a prospective, open-label, non-randomized, multi-center (four sites) phase I/II trial. In study arm A, a maximum of 20 patients will be enrolled. In study arm $\mathrm{B}$, a maximum of 13 patients will be included.

The primary endpoint of this phase I trial is the frequency of a set of predefined TLTs (treatment limiting toxicities), i.e. predefined treatment-limiting skin toxicities or any other toxicity expected to be related to the combination of radiotherapy and TTFields, leading to compliance rate to TTFields therapy of below 50\%, assessed weekly during treatment and up to 4 weeks after the end of radiotherapy. One of the secondary endpoints is the estimation of the delivered cumulative dose distribution over the treatment series for each patient from the $\mathrm{kV}$-image guidance data and comparison with the planned dose distribution. Dose deviations by more than $3.5 \%$ in more than $1 \mathrm{~cm}^{3}$ within the PTV or by more than $5 \%$ in less than $1 \mathrm{~cm}^{3}$ within the PTV will be considered as relevant. The trial will be stopped, if treatment-limiting toxicities occur in three or more patients among the first seven patients in arm $\mathrm{A}$ and two or more patients in arm B, respectively. Otherwise, the recruitment aim is a total of 20 and 13 patients in arms $\mathrm{A}$ and $\mathrm{B}$, respectively. Concomitant and sequential chemotherapy was administered following institutional standards and after interdisciplinary tumor conference consensus.

\section{TTFields-therapy concomitant to radiochemotherapy}

TTFields therapy was initiated approximately one to 2 week before radiotherapy. Patients were trained on handling the device by a support specialist from the manufacturer. In addition, the treating physician advised patients to use TTFields at least $75 \%$ of the time. Positioning of the four transducer arrays consisting of nine electrodes with a diameter of $2.0 \mathrm{~cm}$ on an adhesive tape was planned by the manufacturer according to routine clinical care and to the localization of the tumor. The four arrays were fixed by adhesive tapes on the bald-shaved head. The arrays were changed every three to 4 days and prior to the positioning of new arrays the skin was allowed to be uncovered from the arrays for about four to six hours. Radiotherapy treatment was performed through the transducer arrays by turning off the field generator before irradiation and turning it on immediately after irradiation. At the days of array change, patients were irradiated without the arrays, which were relocated immediately after the radiotherapy. Patients were instructed to shift the arrays from their initial position about half the electrode diameter, i.e. $1 \mathrm{~cm}$, every other change of the transducer arrays.

\section{Radiotherapy}

Radiotherapy planning was based on a $1.5 \mathrm{~mm}$ slice thickness, contrast enhanced computed tomography (CT) scan (Siemens Healthineers, Erlangen, Germany) and fused with the postoperative MRI-scan (Eclipse version 15.5, Varian Medical Systems, Palo Alto, CA, US). The planning CTscan was performed without transducer arrays within the field of view at the day of array change in order to avoid beam hardening artifacts. A mask system consisting of two half-shells was mounted on a base frame that can be separated by a spacer in mm-steps up to $4 \mathrm{~mm}$ (BrainLab, Munich, Germany) allowing the later immobilization of the patient with affixed transducer arrays. At the end of the treatment planning session, patients relocated and fixed the TTF electrode arrays with adhesive tapes following the advice of the planning radiation therapy technologists and physicians. The CT-scan was repeated after immobilizing the patient with the mask system using a spacer.

In the case of normofractionation the maximal dose at the brain stem was planned not to exceed 54 Gy, and 55 Gy at the chiasm and the optic nerves. Using hypofractionation, the maximal dose was limited to $40 \mathrm{~Gy}$ at the brain stem, chiasm and optic nerve. A surface dose $>70 \%$ of the prescribed dose within or up to $6 \mathrm{~mm}$ below the skin on a scalp was defined as high skin dose area according to the 
PriCoTTF phase 1 trial protocol. Patients were classified by the high skin dose area of $\leq$ vs. $>50 \mathrm{~cm}^{2}$ at low or high risk for skin toxicity. In accordance with the EORTCACROP guideline the clinical target volume (CTV) comprised an additional margin of $2 \mathrm{~cm}$ around the GTV delineated on contrast-enhanced postoperative T1-weighted MRI sequences, as well as suspicious hyperintensities on FLAIR (fluid attenuated inversion recovery) sequences with respect to anatomical borders [22]. The planning target volume (PTV) margin was defined by an additional margin of $2-5 \mathrm{~mm}$. A $6 \mathrm{D}$ freedom couch was necessary for a precise image guidance of a PTV margin with $2 \mathrm{~mm}$ margin. Radiotherapy was delivered either conventionally fractionated at $2 \mathrm{~Gy}$ per fraction ad $60 \mathrm{~Gy}$ for patients in arm A or hypofractionated at 2.67 Gy per fraction ad 40.05 Gy, 5 fractions per week, for patients in arm B. Coverage of the PTV should be $\geq 90 \%$ and the D98 of the PTV should be $>95 \%$. Non-coplanar IMRT (Intensity-modulated radiotherapy) or non-coplanar volumetric modulated arc therapy was delivered with $6 \mathrm{MeV}$ photons at a linear accelerator (True Beam STx, Varian Medical Systems, Palo Alto, CA) in order to reduce exit dose in the contralateral brain. The linear accelerator was equipped with six degrees of freedom (6-DoF) couch. For image guidance, a low dose cone beam $\mathrm{CT}(\mathrm{CBCT})$ was acquired before each radiotherapy fraction. The CBCT imaged volume was confined to the skull above the supraorbital line in order to avoid unnecessary irradiation of the eye lens. Clinical dose planning was performed with the Acuros XB calculation algorithm version 15.11.3 available with Aria 15.5. (TPS, Varian Medical Systems, Palo Alto, CA) using non-coplanar, static field and rapid arc IMRT at the Varian TrueBeam linear accelerator system (LINAC, Varian Medical Systems, Palo Alto, CA).

\section{Chemotherapy}

Concomitant and adjuvant chemotherapy are not investigated in this trial and will be administered according to institutional standards and interdisciplinary tumor conference.

\section{Conversion of TTF composition to CT numbers for dose calculation}

For accurate dose calculation with TTFields, the conversion of the electron density of the TTFields relative to water to the CT-numbers in Hounsfield units (HU) had to be established. This was done by two methods: first, by HU estimation from the material composition of the TTFields and second, by measuring the transmission factors behind the TTFields in a plastic water phantom (CIRS, Norfolk, VA, USA) with a Markus chamber (PTW-Freiburg, Freiburg, Germany). Material composition of the TTFields was determined as pertinex, ceramic, and gel. The atomic composition of the material fractions was obtained and the relative volumes and weights were measured resulting in a weighted average HU of 3817 . Secondly, the depth dose curve and the measured doses in the water phantom at depth of 5 to $15 \mathrm{~cm}$ behind the TTFields were compared with that calculated behind a contoured TTField structure using density overrides of the TTField contours by the Acuros XB algorithm version 15.11.3 available with Aria 15.5. (TPS, Varian Medical Systems, Palo Alto, CA). The Acuros $\mathrm{XB}$ algorithm offers the opportunity to assign a HU from 3832 to 7484 as titanium alloys to structures. The leading $\mathrm{HU}$ value fitting best to the measured dose distribution in water behind the TTFields was $3832 \mathrm{HU}$, associated with a mass density of $3.56 \mathrm{~g} / \mathrm{cm}^{3}$. The latter $\mathrm{HU}$ value was used for dose accumulation of the clinical treatment plans with electrodes positioned according to the cone-beam CT's.

\section{Clinical dose calculation and accumulation}

The pre-fraction cone-beam CT (CBCT) data (TrueBeam, Varian Medical Systems, Palo Alto, CA) of the 7 patients in the PriCoTTF trial were used for dosimetric verification and dose accumulation of the non-coplanar intensity modulated radiotherapy (IMRT) treatment plans. Furthermore, it served for geometric analysis of the transducer arrays throughout the course of treatment. The position of the frontal, occipital, right and left-sided array was obtained by assessing the $\mathrm{x}-, \mathrm{y}$ - and $z$-vectors from the low-dose CBCT-scans. The $x-, y$ - and $z$-vectors were determined at the center of one of the nine TTFields of each transducer array throughout all fractions. With an approximate volume of $36 \mathrm{~cm}^{3}$ cleared from beam hardening artifacts, transducer array structures were contoured for each fraction with the "Image Thresholding" tool (TPS Eclipse, Varian Medical Systems, Palo Alto, CA, US). Transducer contours were copied back to the planning CT using the 6 degree of freedom on-line match between the respective cone beam CT and the Planning CT. Consecutive two $\mathrm{mm}$ thick surface contours were delineated in the planning CT till a depth of $10 \mathrm{~mm}$ below the surface using the body contour and excluding all TTF structures from each fraction as the surface. For each fraction with TTFields the actual transducer arrays were integrated into the body contour and overwritten with a density characterized by $3832 \mathrm{HU}$. The individual fraction doses were calculated and accumulated over all fractions using a dose grid of $1.5 \mathrm{~mm}$. Dose accumulation with TTFields over the whole treatment series was performed by adding the doses calculated in the planning CT with TTFields of the respective fraction over all fractions voxel-wise over the body volume. In addition, a difference plot was calculated for the accumulated doses with and without TTFields. Hot spots in different organs and cold spots in the CTV were examined. Dose distributions were calculated with both, a Boltzmann equation solver (Acuros XB, Eclipse version 15.5., Varian Medical 
Systems, Palo Alto, CA) and a Monte Carlo dose calculation engine (Prosoma, version 4.2.). The Monte Carlo dose calculation engine implemented in Prosoma version 4.2. is based on the VMC++ and XVMC- code. It relies on a virtual source model (VSM) of the linear accelerator head. The VSM applies a primary and a secondary photon source as well as an electron contamination source [23], derived from a full Monte Carlo Simulation of the accelerator head with the BEAMnrc MC system [24]. The cut-off electron energy in the Acuros XB algorithm is $200 \mathrm{keV}$ compared to $240 \mathrm{keV}$ in the Monte Carlo algorithm. Altogether $n=10^{9}$ primary histories were calculated per calculated dose distribution with Monte Carlo algorithm with a photon cut-off of $60 \mathrm{keV}$.

\section{Radiobiological models}

The equivalent uniform dose (EUD) for the CTV was obtained according to the clonogen survival model [25]. The fraction of clonogenic tumor cells surviving at $2 \mathrm{~Gy}$ (SF2) was assumed to be 0.5445 [26]. This led to a tumor control probability of $30 \%$ for a tumor with $10^{8}$ clonogens at a total dose of 60 Gy with $2 \mathrm{~Gy} /$ fraction. The estimate of the number of clonogenic tumor cells was obtained from Suit 1992 [27]. In addition, the fractionation sensitivity was characterized by an alpha/beta value of $10 \mathrm{~Gy}$ [26]. The validity of the linear quadratic cell survival model was assumed and the respective surviving fraction of 0.4244 at $2.67 \mathrm{~Gy}$ was obtained. As another parameter of the effectiveness of the delivered radiation dose, the D95 (minimum dose within the $5 \%$ of voxels with the highest dose in the CTV) was evaluated. As a parameter for toxicity in the brain outside the planning target volume, the minimum dose in the $2 \%$ voxels at highest accumulated dose in this structure was analyzed. As skin tolerance increases with decreasing exposed area and is above $60 \mathrm{~Gy}$ with conventional fractionation for areas below $30 \mathrm{~cm}^{2}$, we quantified skin exposure as maximum doses outside areas of $1 \mathrm{~cm}^{2}$ or $25 \mathrm{~cm}^{2}$ at highest doses [28].

\section{Statistical analysis}

Statistical analyses were performed in SAS (version 14.1, SAS Institute, Cary, NC, US). All statistical tests and procedures used in this study are specified along with the results. The procedure PRINCOMP was used for principal component analysis. The procedure NPAR1WAY was performed for computation of the empirical distribution functions (SDF). The Kruskal-Wallis tests and Wilcoxon scores were applied for testing statistically significant differences. The procedure UNIVARIATE was used for calculation of means as well as standard deviations. Kolmogorov-Smirnov test was applied for testing normality. The given $p$-values were 2 -sided, the level of significance was set at $<0.05$.

\section{Results \\ Clinical dose calculation and accumulation}

All seven patients finished concomitant treatment without interruptions or major protocol deviations. Characteristics of the prescribed treatments and details of the applied radiotherapy techniques are given in Table 1. Tables 2 and 3 summarize the dosimetric results from dose accumulation over the treatment series as captured by the cone beam CTs with TTFields and overwriting density of the TTFields with $3832 \mathrm{HU}$ calculated using Acuros XB or Monte Carlo algorithm. As a measure of effectiveness, the D95 for the CTV did not decrease by more than $2 \%$ in comparison to plans without TTFields and was typically below $1 \%$. The same applied for the equivalent uniform dose (EUD). In general, there was a very good agreement of the results by the Acuros $\mathrm{XB}$ and Monte Carlo algorithm. The same is valid for organs at risk as brain outside PTV (Brain - PTV) except for the patient in the fourth row of Tables 2 and 3. In this case a dose difference of $4 \%$ with and without electrodes was observed with the Acuros XB, but only of $1.2 \%$ with the Monte Carlo algorithm. Both algorithms revealed a reduced dose build-up in the first five $2 \mathrm{~mm}$ layers $(0$ $10 \mathrm{~mm}$ ) below the surface of the scalp by the electrodes. The maximum doses outside the "hottest" $1 \mathrm{~cm}^{2}$ at highest dose differed by less than $8.5 \%$ of the prescribed dose according to the Acuros XB and the Monte Carlo algorithm. While Tables 2 and 3 compare the dose volume histograms for the indicated structures with and without electrodes and thereby lose spatial correlation of the voxels with over- and underdosage, Table 4 shows the statistics of the observed voxel-wise dose differences in the first three, superficial $2 \mathrm{~mm}$ layers in the scalp for the dose difference distributions with and without TTFields. In the first $2 \mathrm{~mm}$ layer voxel-wise dose differences of up to $29 \%$ (range 24-29\%) could be seen outside $1 \mathrm{~cm}^{2}$ with the largest dose differences in the build-up region determined by the Monte Carlo algorithm. However, absolute doses normalized to the prescription dose stayed below 100\% outside the "hottest" $1 \mathrm{~cm}^{2}$. In addition, the clinical employed array-renewing schedule leading to irradiations without TTFields every third day adds to a further diminution of these over-dosages by one third. Figure 1 highlights dose difference plots of accumulated dose distributions with and without electrodes.

Principal component analysis (PCA) showed that the first principal position component of the variation of repeated array placement in the direction of the largest variations and the perpendicular second component spanning a tangential plane on the skull had a standard deviation of $1.06 \mathrm{~cm}, 1.23 \mathrm{~cm}, 0.96 \mathrm{~cm}$, and $1.11 \mathrm{~cm}$ for the frontal, 
Table 1 Characteristics of the 7 glioblastoma patients: Tumor localization in the patient; study arm A or B; treatment technique; skin high dose area (surface dose $>70 \%$ of the prescribed dose within or up to $6 \mathrm{~mm}$ below the skin on a scalp area $\mathrm{are}$ of $>50 \mathrm{~cm}$ ); delivered dose; number of fractions with set up cone beam CT; temozolomide

\begin{tabular}{|c|c|c|c|c|c|c|}
\hline $\begin{array}{l}\text { Tumor localization in the patient } \\
\text { identified by the respective row of table }\end{array}$ & $\begin{array}{l}\text { Study arm A } \\
\text { or B }\end{array}$ & $\begin{array}{l}\text { Treatment } \\
\text { technique }\end{array}$ & $\begin{array}{l}\text { Skin high dose } \\
\text { area }\end{array}$ & $\begin{array}{l}\text { Delivered } \\
\text { dose }\end{array}$ & $\begin{array}{l}\text { Number of fractions } \\
\text { with set up cone beam } C T\end{array}$ & Temozolomide \\
\hline Left parietal & A & $\begin{array}{l}\text { Non-coplanar } \\
\text { IMRT }\end{array}$ & $2.4 \mathrm{~cm}^{3} / 12 \mathrm{~cm}^{2}$ & $30 \times 2 \mathrm{~Gy}$ & 28 & $75 \mathrm{mg} / \mathrm{m}^{2}$ \\
\hline Right frontotemporal & A & $\begin{array}{l}\text { Non-coplanar } \\
\text { IMRT }\end{array}$ & $4.4 \mathrm{~cm}^{3} / 22 \mathrm{~cm}^{2}$ & $30 \times 2 \mathrm{~Gy}$ & 28 & $75 \mathrm{mg} / \mathrm{m}^{2}$ \\
\hline Right parietal & B & $\begin{array}{l}\text { Non-coplanar } \\
\text { IMRT }\end{array}$ & $18 \mathrm{~cm}^{3} / 90 \mathrm{~cm}^{2}$ & $\begin{array}{l}15 \times 2.67 \\
\text { Gy }\end{array}$ & 5 & $75 \mathrm{mg} / \mathrm{m}^{2}$ \\
\hline Left frontal & A & $\begin{array}{l}\text { Non-coplanar } \\
\text { arcs }\end{array}$ & $12 \mathrm{~cm}^{3} / 60 \mathrm{~cm}^{2}$ & $30 \times 2 \mathrm{~Gy}$ & 23 & $75 \mathrm{mg} / \mathrm{m}^{2}$ \\
\hline Right frontal & B & $\begin{array}{l}\text { Non-coplanar } \\
\text { arcs }\end{array}$ & $0.4 \mathrm{~cm}^{3} / 2 \mathrm{~cm}^{2}$ & $\begin{array}{l}15 \times 2.67 \\
\text { Gy }\end{array}$ & 15 & $75 \mathrm{mg} / \mathrm{m}^{2}$ \\
\hline Right parietal & A & $\begin{array}{l}\text { Non-coplanar } \\
\text { IMRT }\end{array}$ & $\begin{array}{l}37,1 \mathrm{~cm}^{3} / 185.5 \\
\mathrm{~cm}^{2}\end{array}$ & $30 \times 2$ Gy & 30 & $75 \mathrm{mg} / \mathrm{m}^{2}$ \\
\hline Right frontal & A & $\begin{array}{l}\text { Non-coplanar } \\
\text { IMRT }\end{array}$ & $\begin{array}{l}7.1 \mathrm{~cm}^{3} / 35.5 \\
\mathrm{~cm}^{2}\end{array}$ & $30 \times 2 \mathrm{~Gy}$ & 30 & $75 \mathrm{mg} / \mathrm{m}^{2}$ \\
\hline
\end{tabular}

occipital, left and right arrays for the first and $0.70 \mathrm{~cm}$, $0.71 \mathrm{~cm}, 0.79 \mathrm{~cm}$, and $0.68 \mathrm{~cm}$, respectively for the second principal component (Table 5). Principal component score plots of the length of the first versus second principal component over all fractions and patients is given in Fig. 2 a-d for the occipital, frontal, right and left side arrays along with the $95 \%$ prediction ellipses. The normality of the distribution of the principal components was analyzed by a Kolmogorov-Smirnov test for all arrays. Some deviations were detected (Table 6). The lengths of the first principal vectors did not differ from patient to patient for all arrays $(p>0.8$, Kruskal-Wallis tests).

\section{Discussion}

The present study was designed to investigate, in a predefined way, the dosimetric consequences of TTFields applied during the course of radiochemotherapy. The general aim was to show that radiochemotherapy can be delivered through transducer arrays, both in a practical way and with similar dose distribution as without TTFields. This is of particular importance in view of previous studies that showed that TTFields do not only induce antiproliferative and cytotoxic effects on dividing cells, but also may lead to an enhanced susceptibility and sensitivity to ionizing radiation [21]. Therefore, for the first time, our study shows from a dosimetric point of view that the translation of a combined radiochemoTTF-based therapy into the clinical setting is feasible.

Several novelties are included in this study. First, we estimated the dosimetric effects of transducer arrays on the delivered dose distribution in patients treated with concurrent TTFields and radiation therapy. Second, we used Monto Carlo calculation for estimation of the clinically delivered dose distribution of simultaneous radiotherapy and TTFields in glioblastoma patients. In our study all treatments were performed following the specifications made by the vendor, henceforth all dose analyses were based upon these terms [13].

Table 2 Acuros XB dose calculation: accumulated dose-volume characteristics for the CTV and organs at risk. Each row indicates data from a separate patient

\begin{tabular}{|c|c|c|c|c|}
\hline $\begin{array}{l}\text { CTV D95 without / } \\
\text { with electrodes }\end{array}$ & $\begin{array}{l}\text { CTV EUD without / } \\
\text { with electrodes }\end{array}$ & $\begin{array}{l}\text { Accumulated min surface dose to } \\
\text { the hottest } 1 \mathrm{~cm}^{2} \text { in shells up to } \\
2,4,6,8,10 \mathrm{~mm} \text { without electrodes }\end{array}$ & $\begin{array}{l}\text { Accumulated min surface dose } \\
\text { to the hottest } 1 \mathrm{~cm}^{2} \text { in shells } \\
\text { up to } 2,4,6,8,10 \mathrm{~mm} \text { with electrodes }\end{array}$ & $\begin{array}{l}\text { D2 Brain - PTV } \\
\text { without / with electrodes }\end{array}$ \\
\hline 100.9 / 99.7 & $102.4 / 101.3$ & 69.5 / 80.0 / 88.7 / 94.5 / 102.5 & 77.8 / 82.0 / 87.7 / 96.3 / 100.5 & $83.1 / 82.5$ \\
\hline 97.9 / 99.8 & 100.8 / 99.9 & 87.5 / $99.4 / 100.9$ /100.5 /103.4 & $92.3 / 100.5 / 100.5 / 101.3 / 103.9$ & 88.0 / 87.0 \\
\hline 94.4 / 93.7 & 97.2 / 96.6 & 92.1 / 97.6 / 99.0 / 98.8 / 101.0 & 99.9 / 99.6 / 98.9 / 98.1 / 99.3 & 93.4 / 92.7 \\
\hline 96.8 / 96.3 & $101.0 / 100.4$ & $97.5 / 105.4 / 102.5 / 102.8 / 106.8$ & $101.9 / 108.6 / 102.9 / 102.3 / 105.6$ & 93.8 / 97.8 \\
\hline $98.2 / 97.5$ & $100.1 / 99.4$ & 82.2 / 100.5/ 102.7/ 103.6/ 104.8 & 88.0 / 100.2/ 101.7/ 102.6/ 103.7 & $74.9 / 74.9$ \\
\hline 98.4 / 97.8 & $101.0 / 100.3$ & 93.8 / 103.8/ 104.6/ 105.0/ 106.3 & $102.3 / 104.0 / 104.5 / 104.9 / 106.0$ & $98.7 / 98.0$ \\
\hline 96.6 / 96.4 & 99.6 / 99.5 & 83.8 / 97.3 / 98.6 / 99.7 / 99.9 & 90.3 / 100.3/ 99.5 / 99.7 / 99.5 & 86.2 / 86.0 \\
\hline
\end{tabular}


Table 3 Monte Carlo dose calculation: accumulated dose-volume characteristics for the CTV and organs at risk. Each row indicates data from a separate patient

\begin{tabular}{|c|c|c|c|c|}
\hline $\begin{array}{l}\text { CTV D95 without / } \\
\text { with electrodes }\end{array}$ & $\begin{array}{l}\text { CTV EUD without / } \\
\text { with electrodes }\end{array}$ & $\begin{array}{l}\text { Accumulated min surface dose } \\
\text { to the hottest } 1 \mathrm{~cm} 2 \text { in shells } \\
\text { up to } 2,4,6,8,10 \mathrm{~mm} \text { without } \\
\text { electrodes }\end{array}$ & $\begin{array}{l}\text { Accumulated min surface dose } \\
\text { to the hottest } 1 \mathrm{~cm} 2 \text { in shells } \\
\text { up to } 2,4,6,8,10 \mathrm{~mm} \text { with } \\
\text { electrodes }\end{array}$ & $\begin{array}{l}\text { D2 Brain - PTV without } \\
\text { / with electrodes }\end{array}$ \\
\hline $101.6 / 100.6$ & $103.7 / 102.4$ & 67.8 / 80.3 / $89.1 / 96.6$ / 102.8 & 75.3 / 86.6 / 86.3 / 86.3 / 104.4 & $84.1 / 83.1$ \\
\hline $98.9 / 97.9$ & $102.1 / 101.1$ & 87.5 / 101.7 / 106.9 / 107.5 / 107.0 & 93.4 / 102.4 / $107.1 / 107.0 / 105.9$ & $89.3 / 88.2$ \\
\hline $95.0 / 93.7$ & $98.1 / 97.5$ & 93.5 / 97.4 / 99.6 / 98.9 / 100.5 & 98.1 / 102.0 / 106.2 / 106.8 / 105.8 & $94.8 / 93.8$ \\
\hline $96.3 / 95.8$ & $101.0 / 100.3$ & 93.5 / 103.6/ 101.5 / 101.5 / 105.4 & 99.0 / 110.0 / 101.5 / 109.5 / 109.9 & $93.0 / 92.3$ \\
\hline $97.8 / 97.5$ & $100.6 / 100.0$ & 80.3 / 101.3 / 107.6 / 109.2 / 109.1 & 87.0 / 101.5 / 107.2 / 108.0 / 107.4 & $74.9 / 74.5$ \\
\hline 99.4 / 98.9 & $102.4 / 101.5$ & 96.6 / 102.9 / 104.3 / 104.9 / 106.3 & $100.3 / 106.8 / 110.0 / 110.3 / 110.8$ & 99.4 / 98.8 \\
\hline $100.0 / 99.2$ & $101.8 / 100.8$ & 82.7 / 97.7 / 98.7 / 99.2 / 99.8 & 89.2 / 105.6 / 109.9 / 108.3 / 107.8 & $87.3 / 86.4$ \\
\hline
\end{tabular}

Note: doses are given as relative doses normalized to the prescribed dose; four adjacent scalp+calvaria shells were defined as tissue slices of 2 mm thickness below the surface of the scalp in a depth of $0-2 \mathrm{~mm}, 2-4 \mathrm{~mm}, 4-6 \mathrm{~mm}, 6-8 \mathrm{~mm}$, and 8-10 $\mathrm{mm}$

Previous film dosimetry measurements showed that transducer arrays may increase the dose build-up to 82 , 88 and $98 \%$ of the maximal dose build-up per beam at a depth of $0.4 \mathrm{~mm}$ and incident beam angles on the surface of $90^{\circ}, 45^{\circ}$ and $10^{\circ}$ [29]. In the present study, the maximal voxel-wise dose difference with and without onlying transducer arrays ranged from 24 to $29 \%$ of the prescribed dose observed in the superficial $2 \mathrm{~mm}$ of the scalp outside the $1 \mathrm{~cm}^{2}$ with the largest dose differences. The attenuation of a $6 \mathrm{MeV}$ beam is about $3-4 \%$ at an incidence angle of $90^{\circ}$ to the skin. Back scatter experiments positioning the transducer arrays at the beam exit side of the body showed an enhancement of $23 \%$ of the depth dose at the beam exit side of the body. Li et al. 2018 and Straube et al. 2018 performed treatment planning and TTF dose measurement studies in an Anderson Rando phantom [30, 31]. Either the Acuros XB v11 or the AAA13 algorithm, both implemented in the Eclipse planning system (Varian Medical Systems, Palo Alto, CA, US), were used by Li et al. 2018 and Bender et al. for dose calculation $[29,30]$. Li et al. overwrote the density of the electrodes contoured on the CT of the Anderson RANDO phantom with the highest density allowed by the Acuros XB v11 algorithm, with the density of aluminium, that is lower than that of the TTFields [30]. Straube et al. used the Hounsfield units from a $\mathrm{keV}-\mathrm{CT}$ and $\mathrm{MeV}-\mathrm{CT}$ to estimate the electron densities of the transducer arrays [31]. Straube's group noticed that the Hounsfield units from the keV-CT were at and above the upper limit of measurable values [31]. The $\mathrm{MeV}-\mathrm{CT}$ 's tended to an underestimation of the HU by the electrodes. Li et al. found from the dose calculations that the percentage of the PTV covered by the prescribed dose, decreases by an average of $0.7 \%$ in the 10 scenarios analyzed in the phantom, but could detect dose increases in the scalp only being 2 Gy for the D1cc at maximum as a consequence of the electrodes [30]. The D1cc is the minimum dose in the most exposed 1 $\mathrm{cm}^{3}$, than without the electrodes from the overall dose statistic within the respective structure. In the present study, we found higher dose increases of up to $5.1 \mathrm{~Gy}$ for a conventional fractionated schedule up to $60 \mathrm{~Gy}$ in

Table 4 Monte Carlo calculated dose build up in the scalp: dose differences from the dose differences distribution with - without TTF electrodes in layers of $2 \mathrm{~mm}$ thickness. Note. Dose differences are given as percentage relative doses normalized to the prescription dose

\begin{tabular}{|c|c|c|c|c|c|}
\hline \multicolumn{2}{|c|}{ Scalp shells from 0 to $2 \mathrm{~mm}$ below surface } & \multicolumn{2}{|c|}{ Scalp shells from 2 to $4 \mathrm{~mm}$ below surface } & \multicolumn{2}{|c|}{ Scalp shells from 4 to $6 \mathrm{~mm}$ below surface } \\
\hline $\begin{array}{l}\text { Min dose difference in the } \\
1 \mathrm{~cm}^{2} \text { with the largest dose } \\
\text { differences [\%] }\end{array}$ & $\begin{array}{l}\text { Min dose difference in } \\
\text { the } 25 \mathrm{~cm}^{2} \text { with the } \\
\text { largest dose differences [\%] }\end{array}$ & $\begin{array}{l}\text { Min dose difference in } \\
\text { the } 1 \mathrm{~cm}^{2} \text { with the } \\
\text { largest dose differences } \\
\text { [\%] }\end{array}$ & $\begin{array}{l}\text { Min dose difference } \\
\text { in the } 25 \mathrm{~cm}^{2} \text { with } \\
\text { the largest dose } \\
\text { differences [\%] }\end{array}$ & $\begin{array}{l}\text { Min dose difference } \\
\text { in the } 1 \mathrm{~cm}^{2} \text { with } \\
\text { the largest dose } \\
\text { differences [\%] }\end{array}$ & $\begin{array}{l}\text { Min dose difference } \\
\text { in the } 25 \mathrm{~cm}^{2} \text { with } \\
\text { the largest dose } \\
\text { differences [\%] }\end{array}$ \\
\hline 24.4 & 12.4 & 11.8 & 6.6 & 13.8 & 7.8 \\
\hline 29.0 & 14.1 & 13.4 & 6.1 & 8.0 & 3.3 \\
\hline 26.7 & 11.9 & 17.5 & 7.2 & 17.0 & 9.6 \\
\hline 27.0 & 11.9 & 13.5 & 6.8 & 16.2 & 9.0 \\
\hline 26.3 & 9.0 & 10.7 & 3.0 & 5.3 & 1.0 \\
\hline 27.9 & 15.6 & 13.4 & 9.1 & 14.2 & 10.7 \\
\hline 24.6 & 7.1 & 13.1 & 5.0 & 13.7 & 8.7 \\
\hline
\end{tabular}




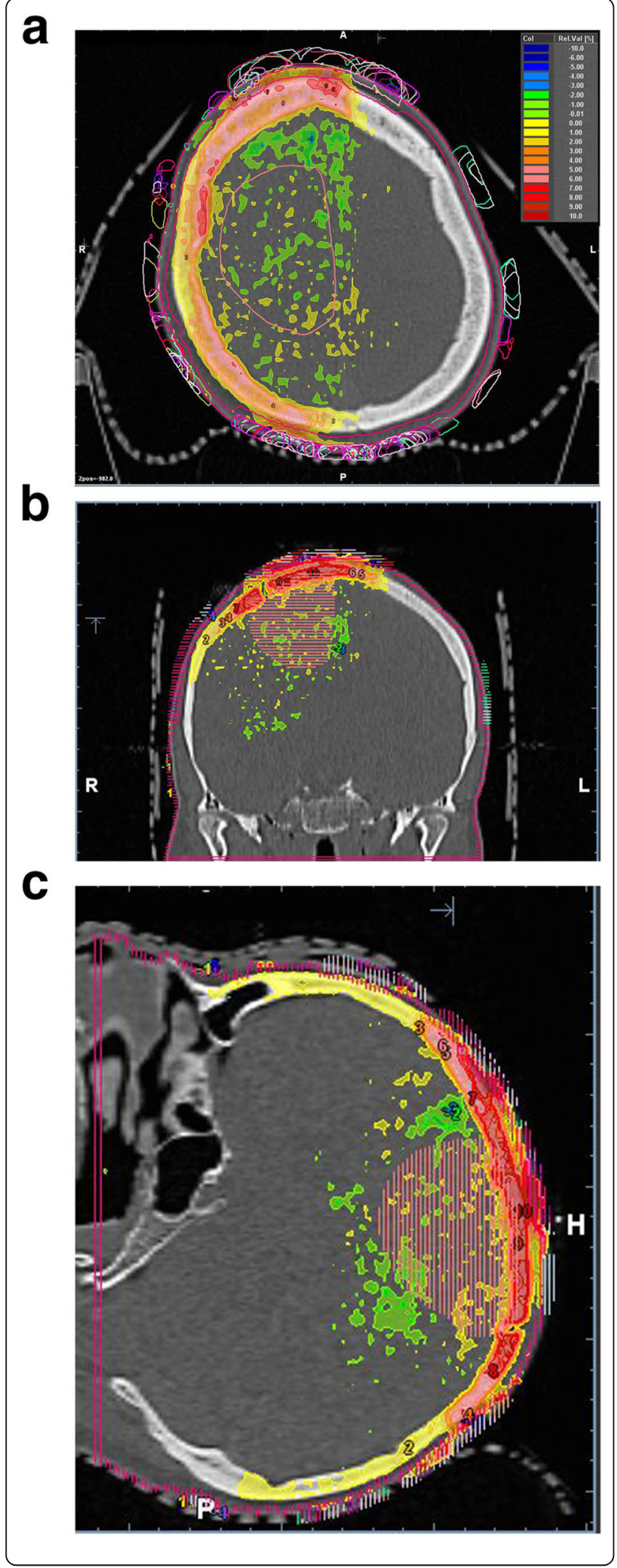

Fig. 1 (a-c) Highlighting dose difference plots of accumulated dose distributions with and without electrodes (a-c): Delineated clinical target volume (right frontoparietal), $2 \mathrm{~mm}$ shell contour and TTF array structures over the whole series (a) right: axial computed tomography; (b) middle: aligned, coronar reconstruction; (c) left: sagittal reconstruction. Dose differences are expressed as percentages of the prescribed dose

the scalp from 0 to $2 \mathrm{~mm}$ below the surface using the maximum dose outside the most exposed $1 \mathrm{~cm}^{2}$ from the dose volume histograms, corresponding to a D0.2cc at a shell thickness of $2 \mathrm{~mm}$, i.e. in smaller hot spots. Straube et al. 2018 found a decrease of $1.1 \%$ in the D98 of the PTV in the analyzed scenarios similar to the results for the CTV in the present study [31]. The previous phantom studies implied that wearing transducer arrays during radiotherapy should not lead to a clinically significant underdosage of the target volume due to the attenuation of the treatment beams [30;31]. However, increased skin doses were noticed. Skin reaction of grade III-IV is the primary endpoint of the PriCoTTF phase I trial. As a primary prophylaxis of severe skin reaction, patients were instructed to remove and replace the transducer arrays at positions differing at maximum one diameter of the electrodes around their original position.

Principal component vector analysis in our study demonstrated that the standard deviation of the position of the center electrode of the anterior and both lateral arrays is $1 \mathrm{~cm}$ in the direction with the largest variation, and $1.4 \mathrm{~cm}$ of the occipital arrays. The vector analysis showed no significant variations from patient to patient. This variation and the fact that at about each third radiation fraction, the transducer arrays were removed during irradiation, led to a marked decrease in the accumulated skin dose and the dose in the subcutaneous tissue below the electrodes.

Furthermore, we compared the clinically used Acuros $\mathrm{XB}$ algorithm and the Prosoma Monte Carlo algorithm used for dose verification in our department. Others have found slight differences between Monte Carlo calculations and the Acuros $\mathrm{XB}$ algorithm in the near the interface of water and high Z-material. Reis et al. 2019 found a good agreement in the depth dose curves in heterogeneous water phantoms with layers of bones with a density of $1.8 \mathrm{~Gy} / \mathrm{cm}^{3}$ [32]. Alhakeem et al. analyzed the performance of the Acuros XB 11.0.31 algorithm in a water phantom containing a steel rood with a density of $7.8 \mathrm{~g} /$ $\mathrm{cm}^{3}$ in comparison to a Monte Carlo simulation and dosimetric measurements [33]. At the distal steel to water interface, the Acuros XB 11.0.31 algorithm underestimated the dose up to 2.8\% [33]. Ojala et al. 2014 compared the agreement of Acuros XB and Monte Carlo dose calculation algorithm point dose measurements in the water phantom [34]. At the distal Ti6A14V alloy hip 
Table 5 Principal component analysis of the placement variations of the transducer arrays: Placement variations along the principal components. Kolmogorov-Smirnov test: Test for comparison of the placement deviations from the overall mean per patient with a normal distribution

\begin{tabular}{|c|c|c|c|c|c|c|}
\hline \multirow[t]{2}{*}{ Array } & \multicolumn{2}{|c|}{ First principal component } & \multicolumn{2}{|c|}{ Second principal component } & \multicolumn{2}{|c|}{ Third principal component } \\
\hline & $\begin{array}{l}\text { Standard deviation } \\
{[\mathrm{cm}]}\end{array}$ & $\begin{array}{l}p \text {-value } \\
\text { Kolmogorov-Smirnov } \\
\text { test }\end{array}$ & $\begin{array}{l}\text { Standard deviation } \\
{[\mathrm{cm}]}\end{array}$ & $\begin{array}{l}p \text {-value } \\
\text { Kolmogorov-Smirnov } \\
\text { test }\end{array}$ & $\begin{array}{l}\text { Standard deviation } \\
{[\mathrm{cm}]}\end{array}$ & $\begin{array}{l}p \text {-value } \\
\text { Kolmogorov-Smirnov } \\
\text { test }\end{array}$ \\
\hline frontal & 1.06 & $<0.01$ & 0.70 & $<0.01$ & 0.31 & $<0.01$ \\
\hline occipital & 1.23 & 0.01 & 0.71 & $<0.01$ & 0.25 & $<0.01$ \\
\hline left & 0.96 & $>0.15$ & 0.79 & $>0.15$ & 0.28 & $<0.01$ \\
\hline right & 1.11 & $>0.15$ & 0.68 & 0.02 & 0.25 & $>0.15$ \\
\hline
\end{tabular}

implant of a density of $4.42 \mathrm{~g} / \mathrm{cm}^{3}$ to water interface, the Acuros XB algorithm underestimated the dose in water near the alloy surface in the shadow of the implant in comparison to the measured and Monte Carlo-calculated doses by up to $5.5 \%$ [34]. Onizuka et al. compared clinical dose distributions for head and neck patients calculated with Monte Carlo and Acuros XB v11 algorithms and found that the Acuros $\mathrm{XB}$ overestimated the dose in the CTV by $3-5 \%$ in comparison to the Monte Carlo simulation technique [35]. Smaller differences between Monte Carlo simulation and the Acuros XB dose calculation algorithm were observed in the superficial dose build-up
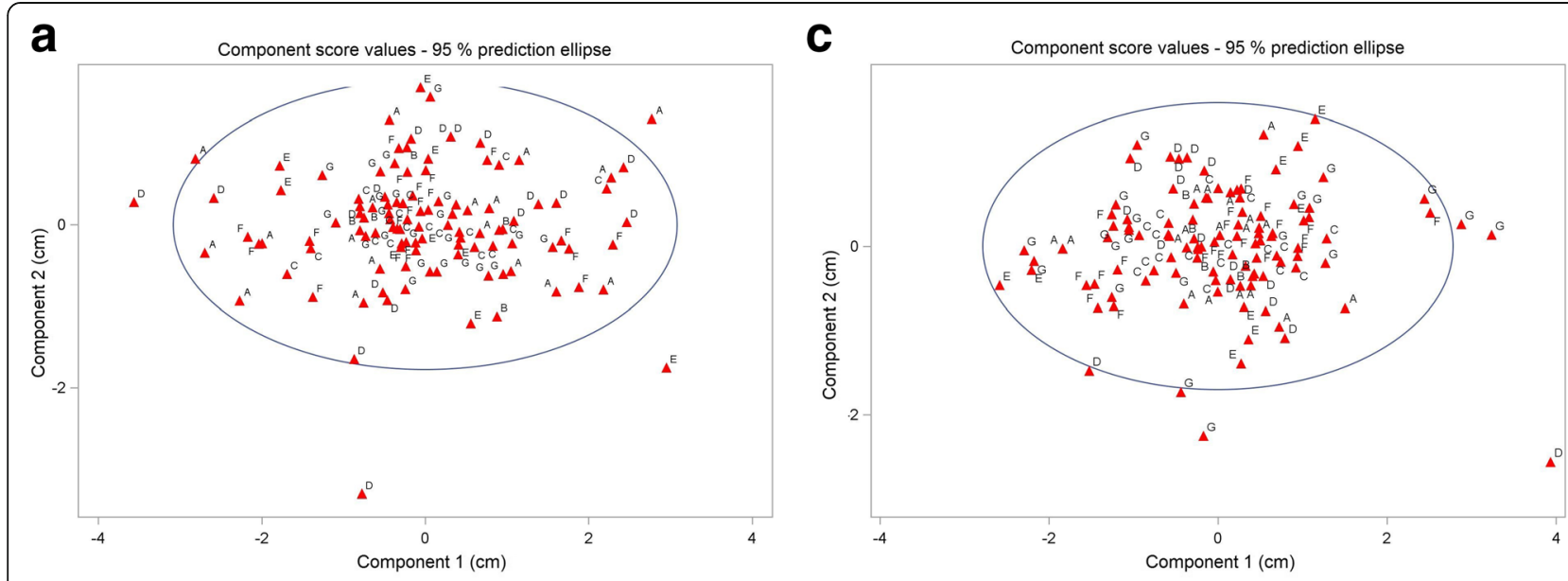

b

d
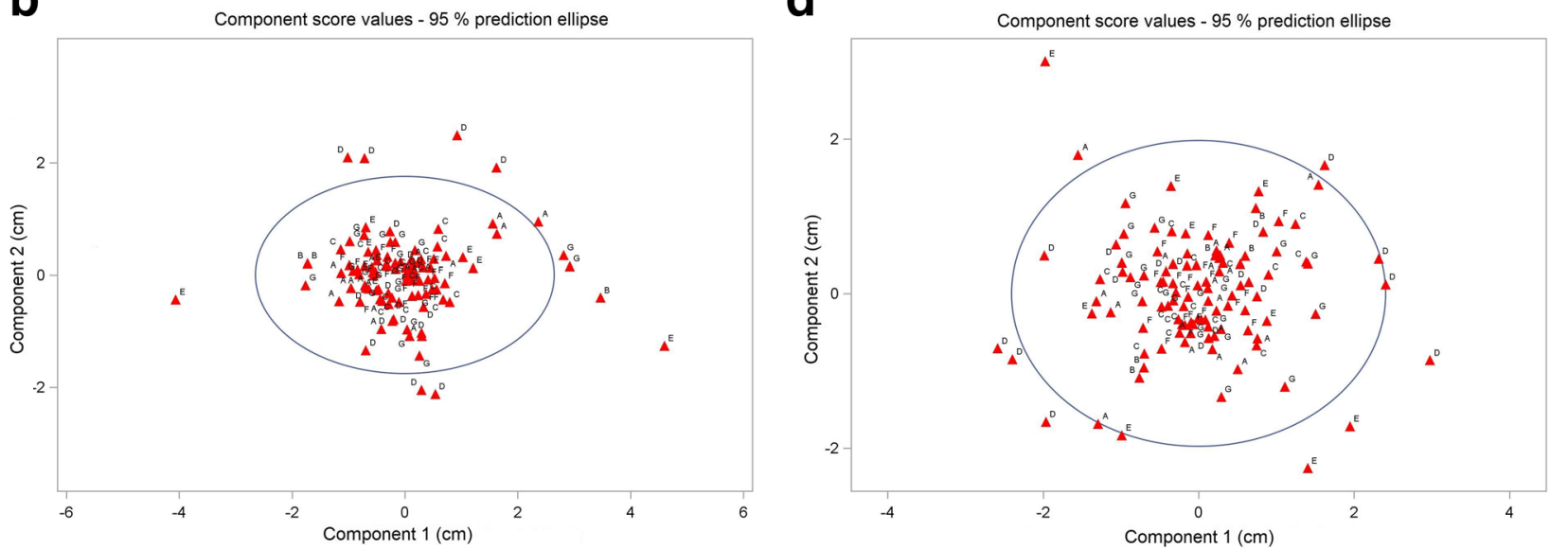

Fig. 2 (a-d) Scatter plots of the first and second principal component length of the respective transducer array position deviation from overall mean position at each fraction for patients a - $\mathrm{g}$ (in $\mathrm{cm}$ ): Data were obtained from pre-fraction cone beam CT's. Data are given together with the 95\% prediction ellipse for a new observation. (a) occipital array; (b) frontal array; (c) right side array; (d) left side array 
Table 6 Principal component analysis of the placement variations of the transducer arrays: coordinates of the principal components. $x, y$, and $z$ are the coordinates of a central electrode of the respective array in the CT-coordinate system relative to the electrode mean position over all fractions. Unit of length is $\mathrm{cm}$

\begin{tabular}{|c|c|c|c|}
\hline Array & $\begin{array}{l}\text { First principal component [cm]/ } \\
\text { Proportion of the variance explained [\%] }\end{array}$ & $\begin{array}{l}\text { Second principal component [cm]/ } \\
\text { Proportion of the variance explained [\%] }\end{array}$ & $\begin{array}{l}\text { Third principal component }[\mathrm{cm}] / \\
\text { Proportion of the variance explained }\end{array}$ \\
\hline frontal & $\begin{array}{c}\mathrm{p} 1=-0.077 x+0.919 y+0.386 z \\
65.7 \%\end{array}$ & $\begin{array}{c}\mathrm{p} 2=0.993 x+0.038 y+0.110 z \\
28.8 \%\end{array}$ & $\begin{array}{c}p 3=-0.087 x-0.392 y+0.916 z \\
5.5 \%\end{array}$ \\
\hline occipital & $\begin{array}{c}p 1=-0.058 x-0.459 y+0.887 z \\
72.8 \%\end{array}$ & $\begin{array}{c}\mathrm{p} 2=0.997 x-0.063 y-0.033 z \\
24.1 \%\end{array}$ & $\begin{array}{c}\mathrm{p} 3=0.041 x+0.886 y+0.461 z \\
3.1 \%\end{array}$ \\
\hline left & $\begin{array}{c}\mathrm{p} 1=0.231 x+0.891 y-0.390 z \\
56.8 \%\end{array}$ & $\begin{array}{c}p 2=-0.305 x+0.447 y+0.841 z \\
38.4 \%\end{array}$ & $\begin{array}{c}\mathrm{p} 3=0.924 x-0.076 y+0.375 z \\
4.8 \%\end{array}$ \\
\hline right & $\begin{array}{c}\mathrm{p} 1=0.017 x+0.993 y-0.119 z \\
70.0 \%\end{array}$ & $\begin{array}{c}p 2=0.345 x+0.1096 y+0.933 z \\
26.3 \%\end{array}$ & $\begin{array}{c}\mathrm{p3}=0.939 x-0.057 y-0.340 z \\
3.7 \%\end{array}$ \\
\hline
\end{tabular}

region. Similar dose differences were observed downstream of the high density structures and in dose buildup regions reported by previous authors [34].

Our results confirm that the dose distribution within the CTV is not clinically significantly compromised by the transducer arrays using multifield, non-coplanar IMRT. Contrary to previous in vivo studies that examined the sequential application of radiotherapy and TTFieldstreatment, our results are the first that showed that radiotherapy with concurrent TTFields-treatment offers a practical treatment option. This is of particular importance considering the fact that several studies reported synergistic effects of concurrent TTF-array treatment and radiotherapy $[16,21]$. We therefore will translate these important results to an already recruiting phase I-trial. If this trial confirms overall safe of the approach and gives first signals for an increased efficacy of radiochemoTTField-therapy, we will explore the approach further in a larger randomized trial that investigates early integration of TTFields concomitant to radiochemotherapy to the conventional sequence of radiochemotherapy followed by chemotherapy and TTField treatment.

\section{Conclusions}

We conclude that dose deviations in the clinical target volume resulting from transducer arrays by which TTFields are applied during conformal radiotherapy treatment are with D95 and EUD differences below 2\% and therefore not clinically relevant. The reduced dose build-up in the skin resulted in a dose increase of below $8.5 \%$ outside the "hottest" $1 \mathrm{~cm}^{2}$ at highest dose and is rated as clinically acceptable.

\section{Abbreviations}

ACROP: Advisory Committee on Radiation Oncology Practice; CBCT: Conebeam computed tomography; CE: Conformité Européenne; CT: Computed tomography; CTV: Clinical target volume; DNA: Deoxyribonucleic acid; EORTC: European Organisation for Research and Treatment of Cancer; EUD: Equivalent uniform dose; Eudamed: European database on medical devices; FDA: U.S. Food and Drug Administration; FLAIR: Fluid attenuated inversion recovery; GBM: Glioblastoma; GTV: Gross tumor volume; HU: Hounsfield unit; IMRT : Intensity modulated radiotherapy; KPS: Karnofsky performance status; PCA: Principal component analysis; PTV: Planning target volume; SF2: Fraction of clonogenic tumor cells surviving at 2 Gy; TMZ: Temozolomide; TLT: Treatment limiting toxicity; TPS: Treatment planning systems; TTFields: Tumor Treating Fields; 6-DoF: Six degrees of freedom

\section{Acknowledgements}

We acknowledge support by the Open Access Publication Fund of the University of Duisburg-Essen.

\section{Clinical relevance statement}

Combined, adjuvant radiochemotherapy and concurrent Tumor Treating Fields offer a practical technique to treat glioblastoma patients.

\section{Authors' contributions}

NG analyzed and interpreted the data and was a major contributor in writing and editing the manuscript. CP has made substantial contributions to the conception of the work. SK has made substantial contributions to the conception of the work. LL has made substantial contributions to the conception of the work. CS has made substantial contributions to the acquisition of the work. WL has made substantial contributions to the acquisition of the work. MN has made substantial contributions to the acquisition of the work. MG has made substantial contributions to the design of the work. BS has made substantial contributions to the conception of the work. VJ has made substantial contributions to the conception of the work. RJ has made substantial contributions to the conception of the work. DP has made substantial contributions to the conception of the work. US has made substantial contributions to the conception of the work. TS has made substantial contributions to the conception of the work. CO has made substantial contributions to the conception of the work. PH has made substantial contributions to the revision of the work. ALG has made substantial contributions to the conception of the work. MS performed the statistical analysis, and was a major contributor in editing the manuscript. MG has made substantial contributions to the conception of the work. YN has made substantial contributions to the acquisition of the work. LL has made substantial contributions to the acquisition of the work. All authors read and approved the final manuscript.

\section{Funding}

The PricoTTF trial is an investigator initiated study supported by Novocure. The present study was not funded by Novocure.

\section{Availability of data and materials Not applicable.}

Ethics approval and consent to participate

The local Ethics Committee approved the trial.

\section{Consent for publication}

Not applicable.

\section{Competing interests}

The authors declare that they have no competing interests. 


\section{Author details}

${ }^{1}$ Department of Radiotherapy, West German Cancer Center, University Hospital Essen, University of Duisburg-Essen, Hufelandstraße 55, 45147 Essen, Germany. ${ }^{2}$ Division of Clinical Neurooncology, Department of Neurology and West German Cancer Center, University Hospital Essen, University of Duisburg-Essen, Hufelandstraße 55, 45147 Essen, Germany. ${ }^{3}$ DKFZ-Division Translational Neurooncology at the West German Cancer Centre (WTZ), German Cancer Consortium (DKTK), Partner Site University Hospital Essen, University of Duisburg-Essen, Duisburg, Germany. ${ }^{4}$ German Cancer Consortium (DKTK), Partner Site University Hospital Essen, Essen, Germany. ${ }^{5}$ Institute of Cell Biology (Cancer Research), University Hospital Essen, Essen, Germany. ${ }^{6}$ Department of Neurosurgery, University Hospital Essen, University of Duisburg-Essen, Essen, Germany. 'Department of Neurology and Wilhelm Sander-NeuroOncology Unit, Regensburg University Hospital, Regensburg, Germany. ${ }^{8}$ Department of Radiation Oncology, University Hospital Freiburg, Freiburg im Breisgau, Germany. ${ }^{9}$ German Cancer Consortium (DKTK) Partner Site University Hospital Freiburg, Heidelberg, Germany.

Received: 27 January 2020 Accepted: 24 March 2020

Published online: 19 April 2020

\section{References}

1. Louis DN, Perry A, Reifenberger G, et al. The 2016 World Health Organization classification of tumors of the central nervous system: a summary. Acta Neuropathol. 2016;131:803-20.

2. Oraiopoulou ME, Tzamali E, Tzedakis G, et al. In vitro/in Silico study on the role of doubling time heterogeneity among primary glioblastoma cell lines. Biomed Res Int. 2017;2017:8569328.

3. Ellingson BM, Nguyen HN, Lai A, et al. Contrast-enhancing tumor growth dynamics of preoperative, treatment-naive human glioblastoma. Cancer. 2016;122:1718-27.

4. Stupp R, Mason WP, van den Bent MJ, et al. European Organisation for Research and Treatment of Cancer Brain Tumor and Radiotherapy Groups; National Cancer Institute of Canada Clinical Trials Group. Radiotherapy plus concomitant and adjuvant temozolomide for glioblastoma. N Engl J Med. 2005;352:987-96 PubMedGoogle ScholarCrossref.

5. Gilbert MR, Dignam JJ, Armstrong TS, et al. A randomized trial of bevacizumab for newly diagnosed glioblastoma. N Engl J Med. 2014;370(8): 699-708 PubMedGoogle ScholarCrossref.

6. Gilbert MR, Wang M, Aldape KD, et al. Dose-dense temozolomide for newly diagnosed glioblastoma: a randomized phase III clinical trial. J ClinOncol. 2013;31:4085-91 PubMedGoogle ScholarCrossref.

7. Chinot OL, Wick W, Mason W, et al. Bevacizumab plus radiotherapytemozolomide for newly diagnosed glioblastoma. N Engl J Med. 2014;370: 709-22.

8. Herrlinger U, Tzaridis T, Mack F, et al. Lomustine-temozolomide combination therapy versus standard temozolomide therapy in patients with newly diagnosed glioblastoma with methylated MGMT promoter (CeTeG/NOA-09): a randomized, open-label, phase 3 trial. Lancet. 2019;393:678-88.

9. Pedicini P, Fiorentino A, Simeon V, et al. Clinical radiobiology of glioblastoma multiforme: estimation of tumor control probability from various radiotherapy fractionation schemes. StrahlentherOnkol. 2014;190:925-32.

10. NCCN Clinical Practice Guidelines in Oncology (NCCN Guidelines ${ }^{\circledR}$ ) Central Nervous System Cancers Version 3.2019 - October 18, 2019. https://www. nccn.org/professionals/physician_gls/pdf/cns.pdf. Accessed 17 Nov 2019.

11. Stupp R, Taillibert S, Kanner A, et al. Effect of tumor-treating fields plus maintenance temozolomide vs maintenance temozolomide alone on survival in patients with glioblastoma: a randomized clinical trial. JAMA. 2017;318:2306-16

12. Stupp R, Wong ET, Kanner AA, et al. Nove TTG-100A versus physician's choice chemotherapy in recurrent gliobalstoma: a randomized phase III trial of a novel treatment modality. Eur J Cancer. 2012;48:2192-202.

13. Novocure. Optune - instructions for use. Document Number: QSD-QR-703. Revision 02. Issue Date: January 2019 Novocure. https://www.optune.com/ Content/pdfs/Optune_IFU_8.5x11.pdf. Accessed 17 Nov 2019.

14. Kirson ED. Alternating electric fields arrest cell proliferation in animal tumor models and human brain tumors. Proc Natl Acad Sci U S A. 2007;104: 10152-1057.

15. Giladi M, Schneiderman RS, Voloshin T, et al. Mitotic spindle disruption by alternating electric fields leads to improper chromosome segregation and mitotic catastrophe in cancer cells. Sci Rep. 2015;5:18046.
16. Giladi M, Munster M, Schneiderman RS, et al. Tumor treating fields (TTFields) delay DNA damage repair following radiation treatment of glioma cells. Radiatoncol. 2017;12:206

17. Kim EH, Song HS, Yoo SH, Yoon M. Tumor treating fields inhibit glioblastoma cell migration, invasion and angiogenesis. Oncotarget. 2016;7: 65125-36. https://doi.org/10.18632/oncotarget.11372.

18. Silginer M, Weller M, Stupp R, Roth P. Biological activity of tumor-treating fields in preclinical glioma models. Cell Death Dis. 2017;8:e2753. https://doi. org/10.1038/cddis.2017.171.

19. Grossman R, Limon D, Bokstein F, Blumenthal D, Harush CB, Ram Z. Tumor Treating Fields combined with radiotherapy and temozolomide for the treatment of newly diagnosed glioblastoma: Final results from a pilot study. Neuro-Oncology. 2019;(Issue Supplement_6):vi23-4. https://doi.org/10.1093/ neuonc/noz175.088.

20. Berkelmann L, Bader A, Meshksar S, et al. Tumour-treating fields (TTFields): investigations on the mechanism of action by electromagnetic exposure of cells in telophase/cytokinesis. Sci Rep. 2019;9:7362.

21. Karanam NK, Srinivasan K, Ding L, et al. Tumor-treating fields elict a conditional vulnerability to ionizing radiation via the downregulation of BRCA1 signaling and reduced DNA double-strand break repair capacity in non-small cell lung cancer cell lines. Cell Death Dis. 2017:8:e2711.

22. Niyazi M, Brada M, Chalmers AJ, et al. ESTRO-ACROP guideline "target delineation of glioblastomas". Radiother Oncol. 2016;118:35-42. https://doi. org/10.1016/j.radonc.2015.12.003.

23. Hoffmann L, Alber M, Söhn M, Elstrøm UV. Validation of the Acuros XB dose calculation algorithm versus Monte Carlo for clinical treatment plans. Med Phys. 2018. https://doi.org/10.1002/mp.13053.

24. Sikora M. Virtual Source Modelling of Photon Beams for Monte Carlo Based Radiation Therapy Treatment Planning, PhD thesis. http://bora.uib.no/ bitstream/handle/1956/5135/Dr.thesis_Marcin\%20Sikora.pdf?sequence= 1 \&isAllowed=y. Accessed 27 Dec 2019.

25. Niemierko A. Reporting and analyzing dose distributions: a concept of equivalent uniform dose. Med Phys. 1997;24:103-10.

26. Stuschke $M$, Budach $V$, Stüben $G$, et al. Heterogeneity in the fractionation sensitivities of human tumor cell lines: studies in a three-dimensional model system. Int J Radiat Oncol Biol Phys. 1995;32:395-408.

27. Suit H, Skates S, Taghian A, et al. Clinical implications of heterogeneity of tumor response to radiation therapy. RadiotherOncol. 1992;25:251-60.

28. Emami B, Lyman J, Brown A, et al. Tolerance of normal tissue to therapeutic irradiation. Int J Radiat Oncol Biol Phys. 1991; 21(1):109-22. https://doi.org/ 10.1016/0360-3016(91)90171-y.

29. Bender E, Kozak K, Howard S, Hayes L, Bayouth J, Robins HI. The effect of Optune ${ }^{T M}$ tumor treating fields transducer arrays on skin radiation dose during radiotherapy. J Clin Neurosci. 2017:42:172-5.

30. Li T, Shukla G, Peng C, Lockamy V, Liu H, Shi W. Dosimetric impact of tumor treating fields device for glioblastoma patients undergoing simultaneous radiation therapy. Front Oncol. 2018;8:51. https://doi.org/10.3389/fonc.2018. 00051.

31. Straube CM, Kampfer OS, et al. Dosimetric impact of tumor treating field (TTField)transducer arrays onto treatment plans for glioblastomas - a planning study. Radiat Oncol. 2018;13:31.

32. Reis CQM, et al. Effects of heterogeneities in dose distributions under nonreference conditions: Monte Carlo simulation vs dose calculation algorithms. Med Dosim. 2019;44:74-82.

33. Alhakeem EY, et al. Comparative evaluation of modern dosimetry techniques near low- and high-density heterogeneities. J ApplClin Med Phys. 2015;16:142-58.

34. Ojala J, Kapanen M, Sipilä $P$, et al. The accuracy of the Acuros XB algorithm for radiation beams traversing a metallic hip implant - comparison with measurements and Monte Carlo simulations. J ApplClin Med Phys. 2014;15: 162-76.

35. Onizuka R, Araki F, Ohno T, et al. Accuracy of dose calculation algorithms for virtual heterogeneous phantoms and intensity-modulated radiation therapy in the head and neck. Radiol Phys tevhnol. 2016;9:77-87.

\section{Publisher's Note}

Springer Nature remains neutral with regard to jurisdictional claims in published maps and institutional affiliations. 